\title{
Charm hadron production in relativistic heavy ion collisions within a quark combination model
}

\author{
Tao Yao, Wei Zhou, and Qu-Bing Xie \\ School of Physics, Shandong University, Jinan, Shandong 250100, P. R. China
}

(Dated: October 25, 2018)

\begin{abstract}
We investigate charm hadron production in relativistic heavy ion collisions with the quark combination model. The $p_{T}$ dependencies of the charm baryon-to-meson ratios such as $\frac{\Lambda_{c}+\bar{\Lambda}_{c}}{D^{0}+\bar{D}^{0}}, \frac{\Lambda_{c}+\bar{\Lambda}_{c}}{D^{+}+D^{-}}$and $\frac{\Lambda_{c}+\bar{\Lambda}_{c}}{D_{s}^{+}+D_{s}^{-}}$in Au+Au collisions at $200 \mathrm{GeV}$ are obtained. The charm baryon enhancement in the intermediate $p_{T}$ range is very prominent, which further, together with the strangeness enhancement, affects the charm hadron ratios. The modified charm hadron ratios lead to a $\sim 17 \%$ increase of the charm cross section given by PHENIX. The dependencies of the charm hadron ratios on energy, centrality, and other parameters are also investigated. Predictions of the charm hadron ratios for the upgrade of RHIC and for LHC are presented.
\end{abstract}

PACS numbers: 25.75.Dw, 12.40.-y, 24.85.+p

\section{INTRODUCTION}

Charm production in high energy heavy ion collisions is one of the hot topics of both theory and experiment. Because of the large mass, charm quarks are believed to be produced mainly via initial gluon fusion in the early stage of relativistic heavy ion collisions [1, 2]. They are also regarded as a unique tool for probing the hot dense matter or quarkgluon plasma(QGP) created in these collisions. For example, through the charm quark energy loss [3], charm flow [4] and $J / \psi$ production (suppression or enhancement) [5, 6, 7], etc., one can learn much about the QGP.

Since the startup of the BNL Relativistic Heavy Ion Collider (RHIC), PHENIX and STAR collaborations have made many measurements on charm production $[8,9,10,11,12,13$, $14,15]$. The binary scaling of the total charm cross section $\sigma_{c \bar{c}}$ has been observed by the two collaborations. However, they give quite different values of the binary scaled charm cross section $\sigma_{c \bar{c}}^{N N}$. There are two important ratios used for obtaining $\sigma_{c \bar{c}}^{N N}$ in the experiments. One is

$$
R_{e / c \bar{c}} \equiv N_{\left(e^{+}+e^{-}\right) / 2} / N_{c \bar{c}}=\sigma_{\left(e^{+}+e^{-}\right) / 2}^{N N} / \sigma_{c \bar{c}}^{N N}
$$

used by PHENIX to convert the multiplicity or cross section of nonphotonic electron into the charm cross section. Here $N_{c \bar{c}}$ is the number of $c \bar{c}$ pairs created in the collisions. Another ratio is

$$
R_{D^{0} / c \bar{c}} \equiv N_{\left(D^{0}+\bar{D}^{0}\right) / 2} / N_{c \bar{c}}=\sigma_{\left(D^{0}+\bar{D}^{0}\right) / 2}^{N N} / \sigma_{c \bar{c}}^{N N}
$$

used by STAR in deriving $\sigma_{c \bar{c}}^{N N}$ from the yield of $D^{0} . R_{e / c \bar{c}}$ [16] and $R_{D^{0} / c \bar{c}}$ are both affected by the charm hadron ratios.

The enhancement of baryon production in the intermediate $p_{T}$ range observed in $\mathrm{Au}+\mathrm{Au}$ reactions at $\mathrm{RHIC}$ suggests strongly a coalescence/recombination (CO/RE) hadronization mechanism [17]. This mechanism can also result in an enhancement of charm baryons, [1] so the charm hadron ratios and $R_{e / c \bar{c}}, R_{D^{0} / c \bar{c}}$ in $\mathrm{Au}+\mathrm{Au}$ collisions should be different from those in $p p(\bar{p})$ reactions or $e^{+} e^{-}$annihilations. Unfortunately, these ratios cannot be obtained through theory calculations model-independently, and it is now difficult to measure them directly in experiments because of the difficulty of charm hadron reconstruction in $\mathrm{Au}+\mathrm{Au}$ collisions. [2] The two key ratios at RHIC are currently from the $p p(\bar{p})$ reactions or $e^{+} e^{-}$annihilations. Then one must ask, how large are the corrections to $\sigma_{c \bar{c}}$ from the two ratios in $\mathrm{Au}+\mathrm{Au}$ collisions, and are the corrections able to account for the discrepancy of $\sigma_{c \bar{c}}^{N N}$ measured by the two collaborations?

A new method is proposed in Ref. [20] to determine $\sigma_{c \bar{c}}^{N N}$ by measuring the spectrum of nonphotonic muon, so the ratio $R_{\mu / c \bar{c}}$, the inclusive branching ratio to muons $\left[\left(\mu^{+}+\mu^{-}\right) / 2\right]$ in $A A$ reactions, is also required.

The charm cross section is one of the most important issues of charm physics in heavy ion collisions. According to Eqs. (1) and (2), the accurate $\sigma_{c \bar{c}}^{N N}$ measurement depends on the accurate ratios $R_{e / c \bar{c}}$ and $R_{D^{0} / c \bar{c}}$, so it is an important issue of charm physics to determine $R_{e / c \bar{c}}, R_{D^{0} / c \bar{c}}, R_{\mu / c \bar{c}}$ and the charm hadron ratios in $A A$ collisions. However, in most combination models, the hadronic "combination function" is necessary to obtain the yield of the hadron. The combination function denotes the probability for (anti)quarks to form a hadron and is determined by the hadron wave function. As the wave functions of charm hadrons are unknown, it is difficult for these models to study the issue quantitatively. In addition, these models do not satisfy the unitarity [21] which is important to the issue as well. By now, only one model [6, 7] has predicted the comprehensive charm hadron ratios at RHIC and LHC within statistical framework. On the other hand, the quark combination model (QCM) we developed satisfies the unitarity and has reproduced the global properties [23, 24, 25, 27] of SU(3) hadrons without the explicit combination functions. Based on the success of QCM, we further extend it to SU(4) flavor symmetry, and QCM is then suitable for studying quantitatively the charm hadron production in heavy ion collisions.

In this paper, we quantitatively study the effects on the 
charm hadron ratios from the baryon enhancement and the strangeness enhancement in $A A$ reactions within the QCM. We find the charm hadron ratios and the key ratios $R_{e / c \bar{c}}, R_{\mu / c \bar{c}}$ are substantially different from those in the $p p(\bar{p})$ reactions or $e^{+} e^{-}$annihilations. Their dependencies on energy, centrality, and some parameters are all investigated. The parameters include the yield ratio of the primary charm vector meson to the pseudoscalar meson $V_{c} / P_{c}$, the yield ratio of primary charm decuplet baryon to octet baryon $D_{c} / O_{c}$, the quark number ratio $N_{\bar{d}} / N_{d}$, the strangeness suppression factor $\lambda_{s}$, and so on. Here $\lambda_{s}$ denotes the number ratio of newly produced strange quarks to $u(d)$ quarks, $\lambda_{s} \equiv \frac{2 N_{s \bar{s}}}{N_{u \bar{u}}+N_{d \bar{d}}}=\frac{N_{\bar{s}}}{N_{\bar{u}}}=\frac{N_{\bar{s}}}{N_{\bar{d}}}$. For the first time in combination or CO/RE mechanism, [3] the extensive charm hadron ratios and the total branching ratios $R_{e / c \bar{c}}$, $R_{D^{0} / c \bar{c}}, R_{\mu / c \bar{c}}$ in $A A$ collisions are predicted at RHIC and LHC. Since the dealings with hadronization are different, it is not surprising that some predictions are different from those in the statistical hadronization model [6, 7]. The predictions can be examined and the different hadronization mechanisms for charm hadrons can be tested in future experiments.

\section{QUARK COMBINATION MODEL}

Our quark combination model (QCM) proposed some time ago [22, 23, 24, 25, 26] demands that quark(s) and/or antiquark(s) which are nearest in rapidity combine into a hadron. It has been shown [23, 24] that such a demand is in agreement with the fundamental requirement of $\mathrm{QCD}$ and uniquely determines the quark combination rule in the hadronization process. QCM has been successfully applied to $e^{+} e^{-}$annihilations and $p p(\bar{p})$ collisions [23, 24, 25]. Recently, we have extended it to the RHIC reactions and have reproduced the global properties of hadrons such as hadronic multiplicities, $p_{T}$ spectra, elliptic flows, and rapidity distributions [27].

QCM is particularly designed for describing the hadronization in the quark $\mathrm{CO} / \mathrm{RE}$ scheme where the properties of the constituent (anti)quarks before hadronization are taken as inputs. In this work, the charm quarks are supposed to be distributed randomly in the light (anti)quark sea after the QGP evolution, and all the (anti)quarks, including charm quarks, combine into hadrons within QCM. All the ground state hadrons, namely, 120-plet baryons and 64-plet mesons for SU(4) quarks, are considered systematically. The higher excited states are not included. The final state hadrons are obtained after all resonances are treated through the decay subroutine in PYTHIA6.4.16 [28]. Some details of QCM can also be seen in Ref. [27].

We determine the parameters $\lambda_{s}$ and $N_{\bar{d}} / N_{d}$ by fitting the two hadron ratios $K^{+} / \pi^{+}$(or $K^{-} / \pi^{-}$) and $\bar{p} / p$. The other hadron ratios can be obtained accordingly with QCM. The parameter $N_{\bar{d}} / N_{d}$, a measure of the net baryon number in QGP,

[3] Note that the combination picture or CO/RE mechanism is the same in all coalescence/recombination/combination models, but the methods to implement the mechanism are different in detail.

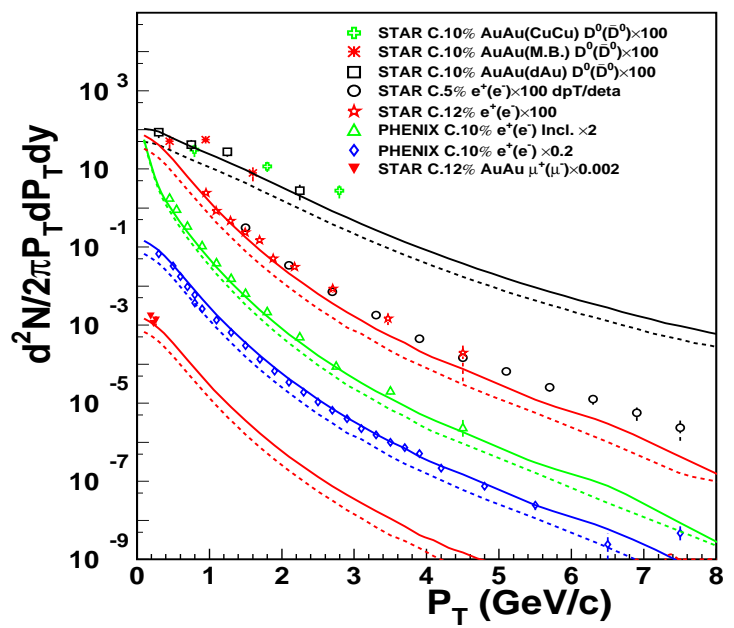

Figure 1: (Color online) Midrapidity $p_{T}$ spectra at $200 \mathrm{GeV}$ for $D^{0}\left(\overline{D^{0}}\right)$ meson, inclusive electron, nonphotonic electron, and muon. The solid (dashed) lines are from $\sigma_{c \bar{c}}^{N N}$ by STAR (PHENIX). $D^{0}$ data tagged with $(\mathrm{CuCu})$ or $(d \mathrm{Au})$ are obtained from $\mathrm{Cu}+\mathrm{Cu}$ or $d+\mathrm{Au}$ collisions based on the binary scaling. Data are from Refs. [9, 10, 11, 12, 13].

is taken as $0.859,0.915$, and 0.927 for $\sqrt{s_{N N}}=62.4,130$, and $200 \mathrm{GeV}$ respectively. Likewise, $\lambda_{s}$ is $0.52,0.48$, and 0.48 for the three RHIC energies, and this is consistent with the data fitting in Ref. [29]. Similar to the same reference, we suppose $\lambda_{s}$ is saturated in $A A$ reactions above $200 \mathrm{GeV}$, that is, QGP created at LHC also has $\lambda_{s}=0.48$. Assuming the parameters $V_{c} / P_{c}$ and $D_{c} / O_{c}$ are universal in relativistic $A A$ reactions, we use 3.0 and 0.5 as their default values in QCM at various energies, respectively. The same default value of $V_{c} / P_{c}$ is also used in PYTHIA.

For simplicity and to reduce the uncertainty of the input, same as Ref. [16], the bottom quarks are not considered in our calculations. According to the pQCD prediction in Ref. [2], the bottom effects on the spectrum of nonphotonic electron are mainly manifested in a higher $p_{T}$ range $\left(p_{T}>4 \mathrm{GeV}\right)$, so we can reliably study the charm hadron multiplicities or ratios while neglecting the bottom contributions. We will not discuss charmonia production here, but will study it in a future paper, as this issue is more complicated and still under debate.

\section{RESULTS AND DISCUSSIONS}

\section{A. $p_{T}$ dependencies of charm baryon-to-meson ratios}

Since most of the data available on charm production in $\mathrm{Au}+\mathrm{Au}$ collisions are at $\sqrt{s_{N N}}=200 \mathrm{GeV}$, we first study charm production in the top central $\mathrm{Au}+\mathrm{Au}$ collisions at this energy. According to the experimental observation of binary scaling of the nonphotonic electron spectra in low $p_{T}$ range, we assume that the total charm cross section or $N_{c \bar{c}}$ in relativistic $A A$ collisions is proportional to the nucleon-nucleon 
collision number $N_{b i n}$. The assumption is consistent with the point that charm quarks are mostly produced via primary hard scattering, and the number of $c \bar{c}$ pairs $N_{c \bar{c}}$, as input in QCM, is given by

$$
N_{c \bar{c}}=\left\langle N_{b i n}\right\rangle \sigma_{c \bar{c}}^{N N} / \sigma_{i n e l}^{p p} .
$$

Here the $\mathrm{Au}+\mathrm{Au}$ charm cross section per nucleon-nucleon collision $\sigma_{c \bar{c}}^{N N}=1.4 \mathrm{mb}$ [9], the $p p$ inelastic cross section $\sigma_{\text {inel }}^{p p}=42 \mathrm{mb}$ and $\left\langle N_{b i n}\right\rangle=1051(939.5)$ [30] are adopted. Then the total $N_{c \bar{c}}$ is about $35(31)$ at $0-5 \%(0-10 \%)$ centrality.

The charm quark spectrum that was input as a normalized form $f_{c}\left(p_{T}\right)=\left(1.0+3.185 p_{T}^{2}\right)^{-2.7} / 0.354$ is extracted by fitting the STAR spectra of $D^{0}\left(\bar{D}^{0}\right)$ and electron. The fitted spectra in Fig. 1 are slightly softer than the STAR data considering no bottom contributions. The solid lines are from the STAR $\sigma_{c \bar{c}}^{N N}$, while the dashed lines, all lower than the data, correspond to the PHENIX $\sigma_{c \bar{c}}^{N N}=0.622 \mathrm{mb}$ [31]. Hereafter, the STAR $\sigma_{c \bar{c}}^{N N} 1.4 \mathrm{mb}$ is used in all calculations at $200 \mathrm{GeV}$.

The $p_{T}$ dependencies of the charm baryon-to-meson ratios $\frac{\Lambda_{c}+\bar{\Lambda}_{c}}{D^{0}+\bar{D}^{0}}, \frac{\Lambda_{c}+\bar{\Lambda}_{c}}{D^{+}+D^{-}}$, and $\frac{\Lambda_{c}+\bar{\Lambda}_{c}}{D_{s}^{+}+D_{s}^{-}}$are plotted in Fig. 2. It is clear to see that the charm baryon enhancement in intermediate $p_{T}$ range is very prominent, which is similar to that of the $p / \pi$ ratio. Note that the peak of $\frac{\Lambda_{c}+\bar{\Lambda}_{c}}{D^{+}+D^{-}}$is even higher than $\frac{\Lambda_{c}+\bar{\Lambda}_{c}}{D_{s}^{+}+D_{s}^{-}}$. This is because the yield of $D_{s}^{+}\left(D_{s}^{-}\right)$is larger than $D^{+}\left(D^{-}\right)$ around $p_{T}=3 \mathrm{GeV} / c$ due to the decay effect. The shapes of the $p_{T}$ dependencies of these ratios are dependent on the $\mathrm{CO} / \mathrm{RE}$ mechanism, decay effect, and the charm quark spectrum obtained from fitting data shown in Fig. 1. Comparing with the data of $p / \pi^{+}[34]$ in the same $\mathrm{Au}+\mathrm{Au}$ collisions, one can see that all peaks broaden and shift to the right because essentially the spectrum of the charm quark is much harder than that of the $u(d)$ quark.

The enhancement can certainly lead to the $R_{A A}$ enhancement of charm baryons $\left(R_{A A} \equiv \frac{d N_{A A} / d y d^{2} p_{T}}{\left\langle N_{b i n} / d N_{p p} / d y d^{2} p_{T}\right.}\right)$ in the same $p_{T}$ region. It can also result in a suppression of nonphotonic lepton spectrum and thus a substantial suppression to its nuclear modification factor $R_{A A}$ in the intermediate $p_{T}$ range [16, 32]. This is just the part of $R_{A A}$ of nonphotonic lepton that arose from the $\mathrm{CO} / \mathrm{RE}$ hadronization process other than the quark energy loss. In the inset of Fig. 2, our calculations also predict a very similar $R_{A A}$ of the nonphotonic muon to that of the nonphotonic electron. [4]

Within the CO/RE hadronization mechanism, we discuss the $R_{A A}$ ordering with strangeness content for charm hadrons in heavy ion collisions. In fact, the $R_{A A}\left(R_{c p}\right)$ ordering with strangeness content for strange hadrons has been observed at RHIC [35, 36], which is a natural result in the CO/RE mechanism for the strangeness enhancement in heavy ion collisions. The effect should also be manifested by the strange charm hadrons such as $R_{A A}$ of $D_{S}^{+}\left(D_{S}^{-}\right)$in comparison with that of nonstrange charm mesons, $R_{A A}$ of $\Xi_{c}$ with respect to that of

[4] The nonphotonic lepton spectra in $p p$ reactions are obtained from PYTHIA6.2 with parameters given in [33].

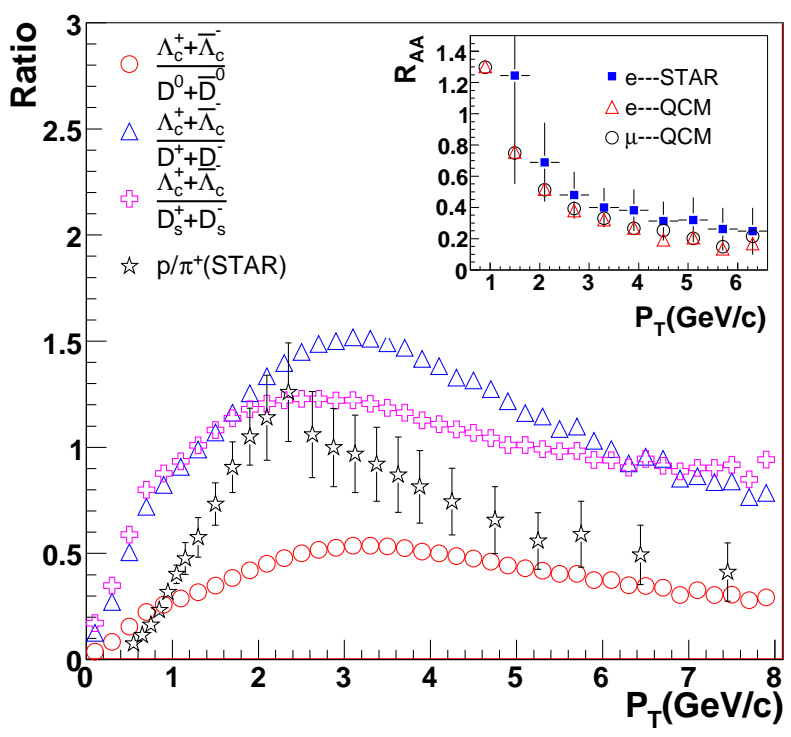

Figure 2: (Color online) Midrapidity $p_{T}$ dependencies for the ratios of charm baryons to mesons at $200 \mathrm{GeV}$. Inset shows $R_{A A}$ of nonphotonic lepton from QCM.

$\Lambda_{c}^{+}$and so on. The $R_{A A}$ ordering with strangeness content for charm hadrons is also a powerful signal for proving the $\mathrm{CO} / \mathrm{RE}$ hadronization mechanism for open charm hadrons. The precise measurement of it in future experiments can help clarify various hadronization mechanisms.

\section{B. Ratios in $A A$ collisions at $200 \mathrm{GeV}$}

As discussed in the last subsection, the CO/RE mechanism results in the charm baryon enhancement which can further affect the charm hadron ratios in relativistic $A A$ reactions. In this subsection, the effects on charm hadron ratios and the three key ratios are quantitatively studied within the QCM.

The rapidity densities of single-charm hadrons and their ratios in $\mathrm{Au}+\mathrm{Au}$ collisions at $200 \mathrm{GeV}$ are listed in Table $\mathrm{We}$ can see that the rapidity densities for charm hadrons are different from those for charm antihadrons because of the net $u / d$ quarks in QGP. The yield of $D^{0}\left(\bar{D}^{0}\right)$ obtained at midrapidity $d N_{\left.\left(D^{0}+\bar{D}^{0}\right) / 2\right)} /\left.d y\right|_{y=0} \approx 3.1$ is in agreement with the STAR data from the $d+\mathrm{Au}$ reactions [9] assuming a binary scaling of $N_{c \bar{c}}$. In Ref. [31], PHENIX modified the PYTHIA default charm hadron ratios, and they obtain $R_{e / c \bar{c}}=9.5 \pm 0.4 \%$ by using $D^{+} / D^{0}=0.45 \pm 0.1, D_{s}^{+} / D^{0}=0.25 \pm 0.1$, and $\Lambda_{c}^{+} / D^{0}=0.1 \pm 0.05$. Obviously, $D_{s}^{+} / D^{+}$from the PHENIX ratios is $0.25 / 0.45 \approx 0.56$. These ratios are apparently different from those in $A A$ collisions listed in Table [ Especially, the ratio $\Lambda_{c}^{+} / D^{0}$ is much enhanced in CO/RE mechanism in $A A$ reactions, as can also be seen in Fig. 2. It is also impressive that the ratio $D_{s}^{+} / D^{+}$is almost equal to 1 , which is much higher than that $(0.56)$ used by PHENIX. The first reason is that $\lambda_{s}$ is taken as 0.48 , much larger than that in $p p$ reactions. The second reason is that $D_{s}^{* \pm}$ totally decays to $D_{s}^{ \pm}$, but only 
about $32.3 \%$ of the $D^{* \pm}$ particles decay to $D^{ \pm}$mesons, and the other $67.7 \%$ decay to $D^{0}\left(\bar{D}^{0}\right)$ mesons. The last reason is that the default value of $V_{c} / P_{c}$ is 3 . However, even if $V_{c} / P_{c}$ equals 1.5 , the ratio $D_{s}^{+} / D^{+} \approx \frac{0.48 \times(1.5+1)}{1.5 \times 32.3 \%+1} \approx 0.81$ is still higher than
0.56 .

Considering the effect from net baryon number, the value of $R_{e / c \bar{c}}$ at midrapidity should be calculated by

$$
\begin{aligned}
R_{e / c \bar{c}} & =\frac{6.87_{-0.28}^{+0.28} \% \times\left(1+\frac{\bar{D}^{0}}{D^{0}}\right)+17.2_{-1.9}^{+1.9} \% \times\left(\frac{D^{+}}{D^{0}}+\frac{D^{-}}{D^{0}}\right)+8_{-5}^{+6} \% \times\left(\frac{D_{s}^{+}}{D^{0}}+\frac{D_{s}^{-}}{D^{0}}\right)+4.5_{-1.7}^{+1.7} \% \times\left(\frac{\Lambda_{c}^{+}}{D^{0}}+\frac{\Lambda_{c}^{-}}{D^{0}}\right)}{1+\frac{\bar{D}^{0}}{D^{0}}+\frac{D^{+}}{D^{0}}+\frac{D^{-}}{D^{0}}+\frac{D_{s}^{+}}{D^{0}}+\frac{D_{s}^{-}}{D^{0}}+\frac{\Lambda_{c}^{+}}{D^{0}}+\frac{\Lambda_{c}^{-}}{D^{0}}} \\
& =\frac{6.87_{-0.28}^{+0.28} \% \times\left(D^{0}+\bar{D}^{0}\right)+17.2_{-1.9}^{+1.9} \% \times\left(D^{+}+D^{-}\right)+8_{-5}^{+6} \% \times\left(D_{s}^{+}+D_{s}^{-}\right)+4.5_{-1.7}^{+1.7} \% \times\left(\Lambda_{c}^{+}+\Lambda_{c}^{-}\right)}{\left(D^{0}+\bar{D}^{0}\right)+\left(D^{+}+D^{-}\right)+\left(D_{s}^{+}+D_{s}^{-}\right)+\left(\Lambda_{c}^{+}+\Lambda_{c}^{-}\right)} .
\end{aligned}
$$

These charm hadron ratios in $\mathrm{Au}+\mathrm{Au}$ collisions give a lower $R_{e / c \bar{c}}=8.48_{-0.90}^{+1.05} \%$ than that used by PHENIX, and it can lead to $\sim 12 \%$ increase of $\sigma_{c \bar{c}}^{N N}$ based on the PHENIX nonphotonic electron data. Here the errors of $R_{e / c \bar{c}}$ are from the branching ratio uncertainties. On the PDGlive web-page, the newest branching ratio for $D^{0}\left(D^{+}\right) \rightarrow e^{+}+$anything is $6.53 \pm 0.17 \%$ $(16.0 \pm 0.4 \%)$, from which the updated $R_{e / c \bar{c}}=8.10_{-0.84}^{+0.99} \%$ results in $\sim 17 \%$ enhancement of $\sigma_{c \bar{c}}^{N N}$ (central value). That is, the ratio $R_{e / c \bar{c}}$ used by PHENIX decreases $\sigma_{c \bar{c}}^{N N}$ by $\sim 17 \%$ and hence enlarges the difference of $\sigma_{c \bar{c}}^{N N}$ between STAR and PHENIX.

Strictly speaking, the ratio obtained by Eq. (4) may be different from the 'real' $R_{e / c \bar{c}}$ ratio, which can be calculated directly in QCM from the $R_{e / c \bar{c}}$ definition of Eq. (1), i.e., from the yield of nonphotonic electrons $N_{\left(e^{+}+e^{-}\right) / 2 \mid y=0}$ and the number of charm quark pairs $\left.N_{c \bar{c}}\right|_{y=0}$ at midrapidity. Note that the denominator of Eq. (4) is smaller than $\left.N_{c \bar{c}}\right|_{y=0}$, and the numerator of Eq. (4) can also be different from $\left.N_{\left(e^{+}+e^{-}\right) / 2}\right|_{y=0}$. The value from Eq. (1) becomes $8.33 \%$, smaller than $8.48 \%$, and this leads to about a $2 \%$ enhancement (systematic error) of $\sigma_{c \bar{c}}^{N N}$. Based on our calculations, such systematic errors for $R_{e / c \bar{c}}$ at the various energies studied in the paper are all smaller than $0.5 \%$, and the resulting enhancements of $\sigma_{c \bar{c}}^{N N}$ are all not more than $5 \%$.

Another ratio used by STAR in Refs. [9, 10] is $R_{D^{0} / c \bar{c}}=$ $0.54 \pm 0.05$ from $e^{+} e^{-}$annihilation data at $91 \mathrm{GeV}$. We can obtain the ratio approximately through the charm hadron ratios at midrapidity, that is,

$$
\begin{aligned}
R_{D^{0} / c \bar{c}} & =\frac{1+\frac{\bar{D}^{0}}{D^{0}}}{1+\frac{\bar{D}^{0}}{D^{0}}+\frac{D^{+}}{D^{0}}+\frac{D^{-}}{D^{0}}+\frac{D_{s}^{+}}{D^{0}}+\frac{D_{s}^{-}}{D^{0}}+\frac{\Lambda_{c}^{+}}{D^{0}}+\frac{\Lambda_{c}^{-}}{D^{0}}} \\
& =\frac{D^{0}+\bar{D}^{0}}{D^{0}+\bar{D}^{0}+D^{+}+D^{-}+D_{s}^{+}+D_{s}^{-}+\Lambda_{c}^{+}+\Lambda_{c}^{-}}(5)
\end{aligned}
$$

Then we have 0.534 in QCM at most central collisions at 200 $\mathrm{GeV}$. The value is by chance very close to that used by STAR and leads to only $\sim 1 \%$ correction of $\sigma_{c \bar{c}}^{N N}$.

If we calculate $R_{D^{0} / c \bar{c}}$ via its definition of Eq. (2), it changes to 0.491 , which means $\sim 8 \%$ enhancement (systematic error) of $\sigma_{c \bar{c}}^{N N}$. As the denominator $\left.N_{c \bar{c}}\right|_{y=0}$ of Eq. (2) is larger than
Table I: Midrapidity densities of open charm hadrons and their ratios for top central collisions at $\sqrt{s_{N N}}=62.4,130,200$ and $5500 \mathrm{GeV}$. $R_{e / c \bar{c}}$ and $R_{D^{0} / c \bar{c}}$ are from Eqs. 44 and (5).

\begin{tabular}{c|c|c|c|c|c|c|c|c}
\hline$d N / d y$ & 62.4 & 130 & 200 & Ratios & 62.4 & 130 & 200 & 5500 \\
\hline$D^{+}$ & 0.217 & 0.532 & 0.938 & $D^{+} / D^{0}$ & 0.317 & 0.317 & 0.317 & 0.318 \\
$D^{-}$ & 0.252 & 0.582 & 1.011 & $D_{s}^{+} / D^{0}$ & 0.336 & 0.309 & 0.310 & 0.311 \\
$D^{0}$ & 0.684 & 1.678 & 2.954 & $\Lambda_{c}^{+} / D^{0}$ & 0.353 & 0.311 & 0.301 & 0.253 \\
$\bar{D}^{0}$ & 0.787 & 1.820 & 3.168 & $\bar{D}^{0} / D^{0}$ & 1.151 & 1.085 & 1.073 & 1.005 \\
$D_{s}^{+}$ & 0.229 & 0.519 & 0.915 & $D^{-} / D^{0}$ & 0.369 & 0.347 & 0.342 & 0.319 \\
$D_{s}^{-}$ & 0.229 & 0.520 & 0.915 & $D_{s}^{-} / D^{0}$ & 0.335 & 0.310 & 0.310 & 0.311 \\
$\Lambda_{c}^{+}$ & 0.241 & 0.522 & 0.890 & $\bar{\Lambda}_{c}^{-} / D^{0}$ & 0.205 & 0.227 & 0.230 & 0.249 \\
$\bar{\Lambda}_{c}^{-}$ & 0.140 & 0.381 & 0.679 & $\Sigma_{c}^{+} / D^{0}$ & 0.081 & 0.071 & 0.069 & 0.057 \\
$\Sigma_{c}^{+}$ & 0.055 & 0.120 & 0.203 & $\Sigma_{c}^{0} / D^{0}$ & 0.076 & 0.066 & 0.064 & 0.052 \\
$\bar{\Sigma}_{c}^{-}$ & 0.032 & 0.087 & 0.155 & $\Xi_{c}^{+} / D^{0}$ & 0.078 & 0.068 & 0.067 & 0.062 \\
$\Sigma_{c}^{0}$ & 0.052 & 0.111 & 0.188 & $\Xi_{c}^{0} / D^{0}$ & 0.079 & 0.068 & 0.067 & 0.062 \\
$\bar{\Sigma}_{c}^{0}$ & 0.029 & 0.080 & 0.142 & $D_{s}^{+} / D^{+}$ & 1.058 & 0.975 & 0.976 & 0.980 \\
$\Xi_{c}^{0}$ & 0.054 & 0.115 & 0.198 & $\bar{\Lambda}_{c}^{-} / \Lambda_{c}^{+}$ & 0.581 & 0.730 & 0.763 & 0.982 \\
$\Xi_{c}^{+}$ & 0.053 & 0.113 & 0.197 & $\Sigma_{c}^{+} / \Lambda_{c}^{+}$ & 0.228 & 0.230 & 0.228 & 0.226 \\
$e_{c}^{ \pm}$ & 0.123 & 0.295 & 0.520 & $R_{e} / c \bar{c}$ & 0.085 & 0.085 & 0.085 & 0.085 \\
$\mu_{c}^{ \pm}$ & 0.125 & 0.298 & 0.526 & $R_{D^{0} / c \bar{c}}$ & 0.529 & 0.534 & 0.534 & 0.532 \\
$N_{c \bar{c}}$ & 1.512 & 3.560 & 6.240 & $R_{\mu} / c \bar{c}$ & 0.083 & 0.084 & 0.084 & 0.086 \\
\hline
\end{tabular}

that of Eq. (5), this kind of systematic error of $R_{D^{0} / c \bar{c}}$ always enlarges $\sigma_{c \bar{c}}^{N N}$. The errors of $R_{D^{0} / c \bar{c}}$ at different energies studied in the paper are not more than $5 \%$, and the corresponding maximal increase of $\sigma_{c \bar{c}}^{N N}$ is $\sim 10 \%$.

Similarly, we calculate $R_{\mu / c \bar{c}}$ by

$$
R_{\mu / c \bar{c}}=\frac{\left.N_{\left(\mu^{+}+\mu^{-}\right) / 2}^{c}\right|_{y=0}}{\left.N_{c \bar{c}}\right|_{y=0}},
$$

and $8.42 \%$ is obtained in midrapidity range.

In short, the effect on $R_{e / c \bar{c}}$ in relativistic $A A$ collisions leads to $\sim 17 \%$ increase of $\sigma_{c \bar{c}}^{N N}$ measured by PHENIX, while the correction of $\sigma_{c \bar{c}}^{N N}$ by STAR from $R_{D^{0} / c \bar{c}}$ is only $\sim 1 \%$. The modifications of $R_{e / c \bar{c}}$ and $R_{D^{0} / c \bar{c}}$ in heavy ion collisions reduce the discrepancy of $\sigma_{c \bar{c}}^{N N}$ between STAR and PHENIX but are not enough to account for it. 
Table II: Dependencies of charm hadron ratios on the parameter $V_{c} / P_{c}$, calculated at midrapidity for central (0-5\%) Au+Au collisions at $200 \mathrm{GeV} . R_{e / c \bar{c}}$ and $R_{D^{0} / c \bar{c}}$ are from Eqs. (4) and (5). The symbol $\nearrow(\searrow)$ denotes increase (decrease) with $V_{c} / P_{c}$.

\begin{tabular}{c|c|c|c|c|c|c|c}
\hline$V_{c} / P_{c}$ & 0.5 & 1.0 & 1.5 & 2.0 & 2.5 & 3.0 & \\
\hline$D^{+} / D^{0}$ & 0.620 & 0.482 & 0.411 & 0.367 & 0.339 & 0.317 & $\searrow$ \\
$D_{s}^{+} / D^{0}$ & 0.381 & 0.348 & 0.332 & 0.322 & 0.315 & 0.310 & $\searrow$ \\
$\Lambda_{c}^{+} / D^{0}$ & 0.379 & 0.343 & 0.326 & 0.314 & 0.307 & 0.301 & $\searrow$ \\
$\bar{D}^{0} / D^{0}$ & 1.072 & 1.071 & 1.071 & 1.073 & 1.074 & 1.073 & \\
$D^{-} / D^{0}$ & 0.670 & 0.520 & 0.443 & 0.396 & 0.366 & 0.342 & $\searrow$ \\
$D_{s}^{-} / D^{0}$ & 0.381 & 0.349 & 0.332 & 0.321 & 0.315 & 0.310 & $\searrow$ \\
$\bar{\Lambda}_{c}^{-} / D^{0}$ & 0.289 & 0.261 & 0.248 & 0.240 & 0.234 & 0.230 & $\searrow$ \\
$R_{e} / c \bar{c}$ & 0.095 & 0.091 & 0.088 & 0.087 & 0.086 & 0.085 & $\searrow$ \\
$R_{D^{0}} / c \bar{c}$ & 0.432 & 0.473 & 0.497 & 0.514 & 0.525 & 0.534 & $\nearrow$ \\
$R_{\mu / c \bar{c}}$ & 0.093 & 0.089 & 0.087 & 0.086 & 0.085 & 0.084 & $\searrow$ \\
\hline
\end{tabular}

\section{Parameter dependencies of the ratios}

Considering the uncertainties of some parameters in $A A$ reactions, especially at LHC energies, it is important to know to which parameters these charm hadron ratios and the three key ratios are sensitive. The parameters $V_{c} / P_{c}, D_{c} / O_{c}, \lambda_{s}, N_{\bar{d}} / N_{d}$, and the charm quark $p_{T}$ spectrum are all possible candidates for affecting these ratios. We will study the effects from these factors respectively.

Based on the data in Refs. [37, 38], there is still much uncertainty regarding $V_{c} / P_{c}$. We vary the value of $V_{c} / P_{c}$ with other conditions unchanged to investigate its effect on these ratios. The results are given in Table II. One can see that the ratio $\bar{D}^{0} / D^{0}$ is almost independent of $V_{c} / P_{c}$, and $R_{D^{0} / c \bar{c}}$ increases with $V_{c} / P_{c}$, whereas the other ratios all decrease monotonically with it. This is because $\sim 67.7 \%$ of $D^{* \pm}$ decays to $D^{0}\left(\bar{D}^{0}\right)$, so more $D^{* \pm}$ will result in a larger $D^{0}$ part of the total charm hadrons. Note that for the $V_{c} / P_{c}$ variance from 0.5 to 3.0, both $R_{e / c \bar{c}}$ and $R_{\mu / c \bar{c}}$ decrease by only $\sim 1 \%$, that is, they are not sensitive to $V_{c} / P_{c}$. The reason is that the lepton branching ratios from charm hadrons are very small; however, the $\sim 1 \%$ variance will lead to $\sim 10 \%$ correction of $\sigma_{c \bar{c}}^{N N}$. In contrast, the increase of $R_{D^{0} / c \bar{c}}$ is $\sim 10 \%$, corresponding to $\sim 20 \%$ variance of $\sigma_{c \bar{c}}^{N N}$.

We also study the effect of the parameter $D_{c} / O_{c}$ on these ratios. The ratios, such as $\Lambda_{c}^{*+} / \Lambda_{c}^{+}$, vary with $D_{c} / O_{c}$ certainly, but all the ratios listed in Table $\amalg$ are hardly affected by it, as the charm decuplet baryons almost totally transform into charm octet baryons.

We further explore the charm ratio variances with the strangeness suppression factor $\lambda_{s}$. The results are listed in Table [II]. The ratios $D_{s}^{+} / D^{0}$ and $D_{s}^{-} / D^{0}$ increase with $\lambda_{s}$ apparently. As the total number of charm pairs is conserved while $\lambda_{s}$ is changing, the more strange charm hadrons there are, and the fewer nonstrange charm hadrons, so $R_{D^{0} / c \bar{c}}$ decreases on the contrary. The increase of $\lambda_{s}$ also results in the relative reduction of the numbers of $u, d(\bar{u}, \bar{d})$ quarks. In the coalescence picture, the effect on the $\Lambda_{c}^{+}(u d c)$ or $\bar{\Lambda}_{c}^{-}(\bar{u} \bar{d} \bar{c})$
Table III: Same as Table $\amalg$ but with the parameter $\lambda_{s}$.

\begin{tabular}{c|c|c|c|c|c|c|c}
\hline$\lambda_{s}$ & 0.38 & 0.43 & 0.48 & 0.53 & 0.58 & 0.63 & \\
\hline$D^{+} / D^{0}$ & 0.318 & 0.318 & 0.317 & 0.318 & 0.317 & 0.317 & \\
$D_{s}^{+} / D^{0}$ & 0.245 & 0.278 & 0.310 & 0.342 & 0.373 & 0.406 & $\nearrow$ \\
$\Lambda_{c}^{+} / D^{0}$ & 0.312 & 0.306 & 0.301 & 0.296 & 0.291 & 0.286 & $\searrow$ \\
$\bar{D}^{0} / D^{0}$ & 1.070 & 1.071 & 1.073 & 1.075 & 1.075 & 1.076 & \\
$D^{-} / D^{0}$ & 0.341 & 0.342 & 0.342 & 0.343 & 0.343 & 0.344 & \\
$D_{s}^{-} / D^{0}$ & 0.246 & 0.278 & 0.310 & 0.342 & 0.374 & 0.406 & $\nearrow$ \\
$\bar{\Lambda}_{c}^{-} / D^{0}$ & 0.239 & 0.235 & 0.230 & 0.225 & 0.221 & 0.217 & $\searrow$ \\
$R_{e} / c \bar{c}$ & 0.085 & 0.085 & 0.085 & 0.085 & 0.085 & 0.085 & \\
$R_{D^{0} / c \bar{c}}$ & 0.549 & 0.541 & 0.534 & 0.527 & 0.519 & 0.512 & $\searrow$ \\
$R_{\mu / c \bar{c}}$ & 0.084 & 0.084 & 0.084 & 0.084 & 0.084 & 0.084 & \\
\hline
\end{tabular}

baryon from the number decrease of $u, d(\bar{u}, \bar{d})$ quarks is much larger than that on the $D^{0}(\bar{u} c)$ meson, so the ratios $\Lambda_{c}^{+} / D^{0}$ and $\bar{\Lambda}_{c}^{-} / D^{0}$ decrease with $\lambda_{s}$ increasing. The ratios $\frac{\bar{D}^{0}}{D^{0}} \sim \frac{u}{\bar{u}}$ and $\frac{D^{-}}{D^{0}} \sim \frac{d}{\bar{u}}$ increase slightly, because the net baryon number or the net $u(d)$ quark number is invariant while the newly born $u \bar{u}(d \bar{d})$ pairs decrease. It is unexpected that $R_{e / c \bar{c}}\left(R_{\mu / c \bar{c}}\right)$ is independent of $\lambda_{s}$. This can be understood from Eq. (4), i.e., $D^{+} / D^{0}, \bar{D}^{0} / D^{0}$, and $D^{-} / D^{0}$ are nearly constant, and the effects from $D_{s}^{+} / D^{0}\left(D_{s}^{-} / D^{0}\right)$ and from $\Lambda_{c}^{+} / D^{0}\left(\bar{\Lambda}_{c}^{-} / D^{0}\right)$ almost cancel out. One also can see that the error of $R_{D^{0} / c \bar{c}}$ and the concomitant error of $\sigma_{c \bar{c}}^{N N}$ from $\lambda_{s}$ is $\sim 2 \%$ and $\sim 4 \%$ assuming the uncertainty of $\lambda_{s}$ is about $30 \%$ due to the $\phi / K^{* 0}$ data at RHIC [39, 40]. Note that the error of $R_{D^{0} / c \bar{c}}$ from $\lambda_{s}$ has no effect on the PHENIX $\sigma_{c \bar{c}}^{N N}$ from $R_{e} / c \bar{c}$.

The dependencies on the net baryon number or $N_{\bar{d}} / N_{d}$ are also investigated in the CO/RE mechanism. Note that the net baryon number or the $u(d)$ quark number decreases with $N_{\bar{d}} / N_{d}$ increasing, so $\frac{D^{-}}{D^{0}} \sim \frac{d}{\bar{u}}$ and $\frac{\bar{D}^{0}}{D^{0}} \sim \frac{u}{\bar{u}}$ decrease with $N_{\bar{d}} / N_{d}$, and $\Lambda_{c}^{+} / D^{0}\left(\bar{\Lambda}_{c}^{-} / D^{0}\right)$ decreases (increases) as the net baryon number decreases. The ratios $\frac{D^{+}}{D^{0}} \sim \frac{\bar{d}}{\bar{u}}, \frac{D_{s}^{+}}{D^{0}} \sim \frac{\bar{s}}{\bar{u}}$, and $\frac{D_{s}^{-}}{D^{0}} \sim \frac{s}{\bar{u}}$ are independent of $N_{\bar{d}} / N_{d}$. Based on Eqs. (4) and (5]), the three key ratios $R_{e / c \bar{c}}, R_{\mu / c \bar{c}}$, and $R_{D^{0} / c \bar{c}}$ are also independent of the net baryon number as the sum of one kind of charm hadron and its antiparticle is conserved, although the yield of the kind of (anti)hadron varies with $N_{\bar{d}} / N_{d}$.

Next we discuss the effect from the charm quark $p_{T}$ spectrum. Because the yields of charm hadrons are integrals of the hadronic $p_{T}$ spectra, their yields and ratios in the whole rapidity range should be fixed with the input $N_{c \bar{c}}$ unchanged. However, considering that the rapidity distributions of the secondary hadrons from decay may be affected by the quark $p_{T}$ spectrum, the midrapidity yields or ratios can also be influenced. To study the effect quantitatively, keeping the input $\sigma_{c \bar{c}}^{N N}=1.4 \mathrm{mb}$ and other parameters unchanged, we replace only the charm quark spectrum by $f_{c}\left(p_{T}\right)=(1.0+$ $\left.3.185 p_{T}^{2}\right)^{-1.0} / 0.88$, which results in much harder spectra of charm hadrons and nonphotonic leptons. These ratios, including $R_{e / c \bar{c}}, R_{\mu / c \bar{c}}$, and $R_{D^{0} / c \bar{c}}$, are all only slightly varied, i.e., they are not sensitive to the quark spectrum. The charm 


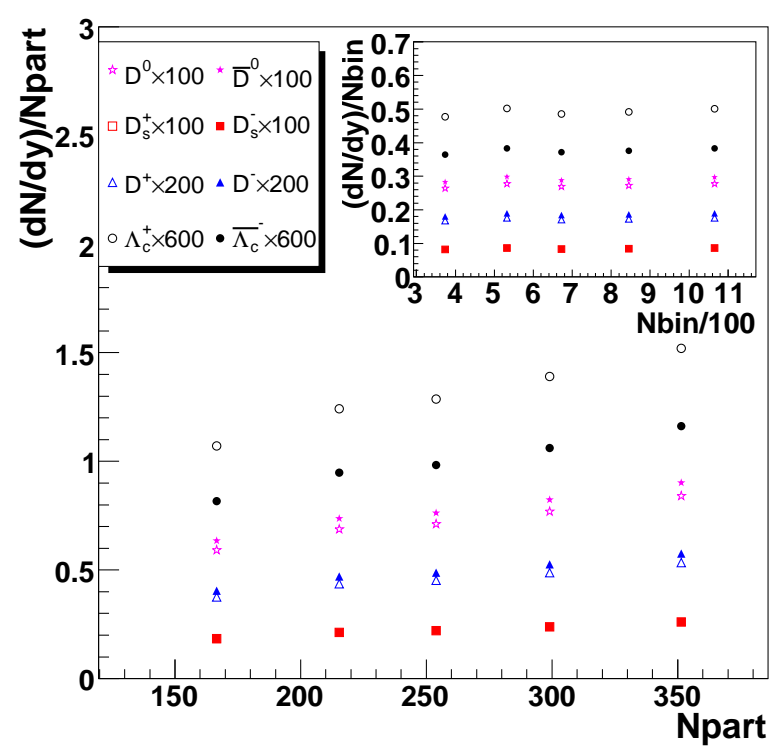

Figure 3: (Color online) Centrality dependencies for midrapidity densities of charm hadrons at $200 \mathrm{GeV}$.

hadron ratios are then determined mainly by the CO/RE mechanism in QCM and some corresponding parameters such as $V_{c} / P_{c}$ other than the $p_{T}$ spectra of quarks. Especially, with this property, if we assume the $\mathrm{CO} / \mathrm{RE}$ hadronization mechanism is universal in relativistic $A A$ reactions, we can give the prediction of charm hadron ratios by QCM without the $p_{T}$ spectra being well determined at LHC energy.

One sees that in the subsection, the ratio $R_{D^{0} / c \bar{c}}$ varies with $V_{c} / P_{c}$ and $\lambda_{s}$, while $R_{e / c \bar{c}}$ and $R_{\mu / c \bar{c}}$ are almost independent of all parameters studied except for a very weak dependence on the parameter $V_{c} / P_{c}$.

\section{Centrality dependencies of the ratios}

We further investigate the centrality dependencies of these ratios at $200 \mathrm{GeV}$ in $\mathrm{Au}+\mathrm{Au}$ collisions. According to the property of little dependence on the charm quark $p_{T}$ spectrum, we can simply deal with the charm quark spectra at various centrality bins by neglecting the differences of radial flows. The numbers of charm quarks at different centrality classes are given by Eq. (3). The parameter $N_{\bar{d}} / N_{d}$ does not vary with the centrality based on the experimental observation of the ratios of antiparticle to particle [41, 42]. The other parameters, such as $\lambda_{s}, D_{c} / O_{c}$, and $V_{c} / P_{c}$ are also assumed to be independent of centrality except for the peripheral collisions. The centrality dependencies of rapidity densities for charm hadrons are given in Fig. 3. One sees that the binary scaled rapidity densities are all independent of $N_{\text {bin }}$. This is consistent with Eq. (3), as in $\mathrm{CO} / \mathrm{RE}$ mechanism the multiplicity of the single-charm hadron $M_{H_{c}}$ satisfies $M_{H_{c}} \propto \frac{d M_{H_{c}}}{d y} \propto N_{c \bar{c}} \propto N_{\text {bin }}$. The participant number scaled rapidity densities increase monotonically with $N_{\text {part }}$ as we have $\frac{d M_{H_{c}}}{N_{\text {part }} d y} \propto N_{\text {part }}^{1 / 3}$ based on Eq. (3) and the approximate relation $N_{\text {bin }} \propto N_{\text {part }}^{4 / 3}$. The result is consistent qualitatively with that of the statistical hadronization model [6] although the input $N_{c \bar{c}}$ is different. Note that the ratio of midrapidity density for single-charm hadrons at a centrality

$$
R_{H_{c}}=\frac{d M_{H_{c}}^{\prime} / d y / N_{\text {part }}}{d M_{H_{c}} / d y / N_{\text {part }}}=\frac{d M_{H_{c}}^{\prime} / d y / N_{\text {bin }}}{d M_{H_{c}} / d y / N_{b i n}}=\frac{d M_{H_{c}}^{\prime} / d y / N_{c \bar{c}}}{d M_{H_{c}} / d y / N_{c \bar{c}}}
$$

is irrelevant to the $N_{\text {part }}, N_{\text {bin }}$ or $N_{c \bar{c}}$, so the ratios of singlecharm hadrons and the three key ratios are all independent of the centrality or $N_{c \bar{c}}$ as long as Eq. (3) is held and those parameters are independent of the centrality.

\section{E. Energy dependencies of the ratios}

To explore the energy dependencies of the charm hadron ratios and the three key ratios, furthermore, we study them at 130 and $62.4 \mathrm{GeV}$ center-of-mass energies at most central $\mathrm{Au}+\mathrm{Au}$ collisions and 5.5 TeV LHC energy for $\mathrm{Pb}+\mathrm{Pb}$ collisions. $\sigma_{c \bar{c}}^{N N}=750,285$ and about $20000 \mu \mathrm{b}$ at the three energies are obtained from the next-to-leading (NLO) pQCD calculations with $\mu_{R}$ equal to $m_{c}$ [43] (or see Fig. 1 in Ref. [44]). We also use $\left\langle N_{b i n}\right\rangle=965$ [45], 904.3 [46] and $1303(0-10 \%$ centrality) [47]. Then we get $N_{c \bar{c}} \approx 18,7$, and 434 as inputs assuming $\sigma_{\text {inel }}^{p p}=41,36$ and $60 \mathrm{mb}$, respectively.

Note that Eq. (7) is still valid at different energies, that is, within the CO/RE framework, the ratios of single-charm hadrons and the three key ratios are independent of charm cross section. This is important to the study of these ratios at various energies, especially at LHC energy, as by now, the theory predictions of $\sigma_{c \bar{c}}$ at LHC still have large uncertainties [43, 48, 49, 50]. Assuming the CO/RE mechanism and the default values of $D_{c} / O_{c}$ and $V_{c} / P_{c}$ are universal in relativistic heavy ion collisions, the other factors that affect these ratios at various energies are mainly $\lambda_{s}$ and the net baryon number.

Using $\lambda_{s}=0.48$ and $\bar{p} / p=0.98$ [51], the charm hadron ratios in top central $\mathrm{Pb}+\mathrm{Pb}$ collisions at $5.5 \mathrm{TeV}$ are obtained. [5] The LHC predictions, together with those at 130 and 62.4 $\mathrm{GeV}$, are all listed in Table 1 , where one can see that $D_{s}^{+} / D^{0}$, $D_{s}^{-} / D^{0}, D_{s}^{+} / D^{+}$, and $R_{D^{0} / c \bar{c}}$ vary mainly with $\lambda_{s}$ at different energies. As the net baryon number decreases with the increasing incident energy, the ratios $\Lambda_{c}^{+} / D^{0}, \Sigma_{c}^{+} / D^{0}, \Sigma_{c}^{0} / D^{0}$, $\Xi_{c}^{+} / D^{0}, \Xi_{c}^{0} / D^{0}, \bar{D}^{0} / D^{0}$, and $D^{-} / D^{0}$ decrease, while $\bar{\Lambda}_{c}^{-} / D^{0}$ and $\bar{\Lambda}_{c}^{-} / \Lambda_{c}^{+}$increase. The other ratios $D^{+} / D^{0}, \Sigma_{c}^{+} / \Lambda_{c}^{+}, R_{e} / c \bar{c}$, and $R_{\mu / c \bar{c}}$ are almost independent of the energy.

In this subsection, one can see that $R_{D^{0} / c \bar{c}}$ depends on the incident energy due to its $\lambda_{s}$ dependency, whereas $R_{e / c \bar{c}}$ and $R_{\mu / c \bar{c}}$ are independent of the energy. The ratios $R_{e / c \bar{c}}$ and $R_{\mu / c \bar{c}}$ are suitable quantities in relativistic heavy ion collisions for determining the charm cross sections at different energies.

[5] The results with input of $\bar{p} / p=0.948$ [52] are very close to those from $\bar{p} / p=0.98$. 


\section{SUMMARY}

By using the QCM, we study the charm hadron production in top central $\mathrm{Au}+\mathrm{Au}$ collisions at $\sqrt{s_{N N}}=200 \mathrm{GeV}$. The $p_{T}$ dependencies of the charm baryon-to-meson ratios, such as $\frac{\Lambda_{c}+\bar{\Lambda}_{c}}{D^{0}+\bar{D}^{0}}, \frac{\Lambda_{c}+\bar{\Lambda}_{c}}{D^{+}+D^{-}}$, and $\frac{\Lambda_{c}+\bar{\Lambda}_{c}}{D_{s}^{+}+D_{s}^{-}}$are obtained. One can see that the charm baryon enhancement in intermediate $p_{T}$ range, similar to that of the $p / \pi$ ratio, is very prominent. The shape differences from that of the $\mathrm{p} / \pi$ ratio are mainly from the hard charm quark spectrum. The $R_{A A}$ ordering with strangeness content for charm hadrons is discussed, and it can be regarded as a powerful signal for proving the $\mathrm{CO} / \mathrm{RE}$ hadronization mechanism for open charm hadrons. The midrapidity densities of the single-charm hadrons and their ratios at $200 \mathrm{GeV}$ are calculated. These ratios in $A A$ collisions, including the key ratios $R_{e / c \bar{c}}$ and $R_{\mu / c \bar{c}}$, are apparently different from those in $p p(\bar{p})$ reactions or $e^{+} e^{-}$annihilations because of the charm baryon enhancement and the strangeness enhancement. With the newest branching ratios from PDG, the modified charm hadron ratios lead to $\mathrm{a} \sim 17 \%$ increase of the central value of $\sigma_{c \bar{c}}^{N N}$ measured by PHENIX. However, this correction is not enough to account for the discrepancy of $\sigma_{c \bar{c}}^{N N}$ between STAR and PHENIX. Considering the uncertainties of some parame- ters, we systematically explore the charm ratio dependencies on various parameters. Assuming the CO/RE hadronization mechanism is universal in relativistic heavy ion collisions, we further investigate the energy dependencies of these ratios, and reveal that these ratios are mainly dependent on $\lambda_{s}$ and the net baryon number but not on the charm cross section. The predictions of charm hadron ratios at $\sqrt{s_{N N}}=62.4,130$, and $200 \mathrm{GeV}$ for the upgrade of RHIC and at $5.5 \mathrm{TeV}$ for LHC are given. These ratios are important for the precise measurement of $\sigma_{c \bar{c}}^{N N}$ in the future, and the $\mathrm{CO} / \mathrm{RE}$ hadronization mechanism for charm hadrons can be tested at RHIC and LHC.

\section{Acknowledgments}

The authors thank Lie-Wen Chen, Che-Ming Ko, Fu-Qiang Wang, Qun Wang, and the colleagues of THPP in Shandong University for helpful discussions. Special thanks go to Chen Zhong (SIAP) for rendering some data adopted in the manuscript. The work is supported in part by National Natural Science Foundation of China (NSFC) under grant Nos. 10475049, 10775089, 10775090.
[1] Z. W. Lin and M. Gyulassy, Phys. Rev. C 51, 2177 (1995); 52, 440(E) (1995).

[2] M. Cacciari, P. Nason, and R. Vogt, Phys. Rev. Lett. 95, 122001 (2005).

[3] Y. L. Dokshitzer and D. E. Kharzeev, Phys. Lett. B519, 199 (2001); M. Djordjevic and M. Gyulassy, ibid. B560, 37 (2003); B. W. Zhang, E. Wang, and X. N. Wang, Phys. Rev. Lett. 93, 072301 (2004); N. Armesto, A. Dainese, C. A. Salgado, and U. A. Wiedemann, Phys. Rev. D 71, 054027 (2005).

[4] N. Xu and Z. B. Xu, Nucl. Phys. A715, 587 (2003); S. Batsouli, S. Kelly, M. Gyulassy and J. L. Nagle, Phys. Lett. B557, 26 (2003); Z. W. Lin and D. Molnar, Phys. Rev. C 68, 044901 (2003); V. Greco, C. M. Ko and R. Rapp, Phys. Lett. B595, 202 (2004); G. D. Moore and D. Teaney, Phys. Rev. C 71, 064904 (2005); R. Rapp and H. van Hees, J. Phys. G 32, S351 (2006).

[5] T. Matsui and H. Satz, Phys. Lett. B178, 416 (1986); R. L. Thews, M. Schroedter and J. Rafelski, Phys. Rev. C 63, 054905 (2001); L. Grandchamp, R. Rapp and G. E. Brown, Phys. Rev. Lett. 92, 212301 (2004); L. Yan, P. Zhuang and N. Xu, ibid. 97, 232301 (2006); B. Zhang, C. M. Ko, B. A. Li, Z. W. Lin and S. Pal, Phys. Rev. C 65, 054909 (2002); E. L. Bratkovskaya, W. Cassing and H. Stoecker, ibid. 67, 054905 (2003).

[6] A. Andronic, P. Braun-Munzinger, K. Redlich and J. Stachel, Phys. Lett. B571, 36 (2003).

[7] A. Andronic, P. Braun-Munzinger, K. Redlich and J. Stachel, Nucl. Phys. A789, 334 (2007).

[8] A. Tai (STAR Collaboration), J. Phys. G 30, S809 (2004).

[9] J. Adams et al. (STAR Collaboration), Phys. Rev. Lett. 94, 062301 (2005).

[10] S. Baumgart (STAR Collaboration), arXiv:0709.4223

[11] C. Zhong (STAR Collaboration), J. Phys. G 34, S741 (2007).

[12] B. I. Abelev et al. (STAR Collaboration), Phys. Rev. Lett. 98,
192301 (2007).

[13] A. Adare et al. (PHENIX Collaboration), Phys. Rev. Lett. 98, 232301 (2007); 98, 172301 (2007); S.S. Adler, et al. (PHENIX Collaboration), ibid. 96, 032301 (2006); 94, 082301 (2005); K. Adcox et al. (PHENIX Collaboration), ibid. 88192303 (2002).

[14] S. S. Adler et al. (PHENIX Collaboration), Phys. Rev. Lett. 96, 032001 (2006); A. Adare et al. (PHENIX Collaboration), ibid. 97252002 (2006).

[15] S. S. Adler et al. (PHENIX Collaboration), Phys. Rev. C 72, 024901 (2005).

[16] P. R. Sorensen and X. Dong, Phys. Rev. C 74, 024902 (2006).

[17] R. C. Hwa and C. B. Yang, Phys. Rev. C 67, 034902 (2003); 70, 024905 (2004); V. Greco, C. M. Ko and P. Levai, Phys. Rev. Lett. 90, 202302 (2003); Phys. Rev. C 68, 034904 (2003); R. J. Fries, B. Muller, C. Nonaka and S. A. Bass, Phys. Rev. Lett. 90, 202303 (2003); Phys. Rev. C 68, 044902 (2003).

[18] S. H. Lee, K. Ohnishi, S. Yasui, I. K. Yoo and C. M. Ko, Phys. Rev. Lett. 100, 222301 (2008).

[19] K. Schweda (STAR Collaboration), Nucl. Phys. A774, 907 (2006); A. Taketani (PHENIX Collaboration), ibid. A774, 911 (2006).

[20] H. D. Liu, Y. F. Zhang, C. Zhong and Z. B. Xu, Phys. Lett. B639, 441 (2006).

[21] D. Molnar and S. A. Voloshin, Phys. Rev. Lett. 91, 092301 (2003); D. Molnar, J. Phys. G 30, S235 (2004); C. B. Yang, ibid. 32, L11 (2006).

[22] Q. B. Xie and Z. T. Liang, In “Jinan 1987, Proceedings, Multiparticle production”, edited by R. C. Hwa and Q. B. Xie, (World Scientific, Singapore, 1987) p. 469-496.

[23] Q. B. Xie and X. M. Liu, Phys. Rev. D 38, 2169 (1988).

[24] Q. B. Xie, in "19th International Symposium on Multiparticle Dynamics 1988”, edited by D. Schiff and J.Tran Thanh Van, (Editions Frontières, Gif-sur-Yvette, France, and World Scien- 
tific, Singapore, 1988), p. 369.

[25] Z. T. Liang and Q. B. Xie, Phys. Rev. D 43, 751 (1991); Q. Wang and Q. B. Xie, J. Phys. G 21, 897 (1995); J. Q. Zhao, Q. Wang and Q. B. Xie, Sci. China Ser. A 38, 1474 (1995); Q. Wang, Z. G. Si and Q. B. Xie, Int. J. Mod. Phys. A 11, 5203 (1996); Z. G. Si, Q. B. Xie and Q. Wang, Commun. Theor. Phys. 28, 85 (1997).

[26] Q. Wang, Q. B. Xie and Z. G. Si, Phys. Lett. B388, 346 (1996); Q. Wang and Q. B. Xie, Phys. Rev. D 52, 1469 (1995); Q. Wang, G. Gustafson and Q. B. Xie, ibid. 62, 054004 (2000); Q. Wang, G. Gustafson, Y. Jin and Q. B. Xie, ibid. 64, 012006 (2001).

[27] F. L. Shao, Q. B. Xie and Q. Wang, Phys. Rev. C 71, 044903 (2005); F. L. Shao, T. Yao and Q. B. Xie, ibid. 75, 034904 (2007); T. Yao, Q. B. Xie and F. L. Shao, Chinese Physics C 32(05), 356 (2008); J. Song, F. L. Shao, Q. B. Xie, Y. F. Wang and D. M. Wei, arXiv:0801.0918 [hep-ph].

[28] T. Sjostrand, P. Eden, C. Friberg, L. Lonnblad, G. Miu, S. Mrenna and E. Norrbin, Comput. Phys. Commun. 135, 238 (2001).

[29] F. Becattini and J. Manninen, J. Phys. G 35, 104013 (2008)

[30] J. Adams et al. (STAR Collaboration), Phys. Rev. C 70, 054907 (2004).

[31] S. S. Adler et al. (PHENIX Collaboration), Phys. Rev. Lett. 94, 082301 (2005).

[32] G. Martinez-Garcia, S. Gadrat and P. Crochet, Phys. Lett. B663, 55 (2008).

[33] X. Y. Lin, arXiv:hep-ph/0602067.

[34] B. I. Abelev et al. (STAR Collaboration), Phys. Rev. Lett. 97, 152301 (2006).
[35] S. Salur (STAR Collaboration), Nucl. Phys. A774, 657 (2006).

[36] B. I. Abelev et al. (STAR Collaboration), Phys. Rev. Lett. 99, 112301 (2007).

[37] L. Gladilin, arXiv:hep-ex/9912064

[38] W. M. Yao et al. (Particle Data Group), J. Phys. G 33, 1 (2006).

[39] C. Adler et al. (STAR Collaboration), Phys. Rev. C 66, 061901 (2002).

[40] J. Adams et al. (STAR Collaboration), Phys. Rev. C 71, 064902 (2005).

[41] S. S. Adler et al. (PHENIX Collaboration), Phys. Rev. C 69, 034909 (2004).

[42] B. Alver et al., Phys. Rev. C 77, 061901 (2008)

[43] R. Vogt (Hard Probe Collaboration), Int. J. Mod. Phys. E 12, $211(2003)$.

[44] Z. Xu, J. Phys. G 32, S309 (2006).

[45] C. Adler et al. (STAR Collaboration), Phys. Rev. Lett. 89, 202301 (2002).

[46] J. Adams et al. (STAR Collaboration), Phys. Rev. C 73, 034906 (2006).

[47] N. Armesto, J. Phys. G 35, 104042 (2008)

[48] R. Vogt, J. Phys. G 31, S773 (2005).

[49] B. W. Zhang, C. M. Ko and W. Liu, Phys. Rev. C 77, 024901 (2008).

[50] W. Liu and R. J. Fries, Phys. Rev. C 78, 037902 (2008)

[51] K. J. Eskola, H. Honkanen, H. Niemi, P. V. Ruuskanen and S. S. Rasanen, Phys. Rev. C 72, 044904 (2005).

[52] A. Andronic, P. Braun-Munzinger and J. Stachel, Nucl. Phys. A772, 167 (2006). 